\title{
Pericardial Rupture and Chronic Subluxation of the Heart: A Case Report
}

\author{
Pedram Rajabifard, Anurag Agarwal, Peter Skillington \\ Department of Cardiothoracic Surgery, Royal Melbourne Hospital, Victoria, Australia \\ Email: pedram.r0@gmail.com
}

How to cite this paper: Rajabifard, P., Agarwal, A. and Skillington, P. (2021) Pericardial Rupture and Chronic Subluxation of the Heart: A Case Report. World Journal of Cardiovascular Surgery, 11, 75-81.

https://doi.org/10.4236/wjcs.2021.119011

Received: August 11, 2021

Accepted: September 26, 2021

Published: September 29, 2021

Copyright $\odot 2021$ by author(s) and Scientific Research Publishing Inc. This work is licensed under the Creative Commons Attribution International License (CC BY 4.0).

http://creativecommons.org/licenses/by/4.0/

\section{Open Access}

\begin{abstract}
Background: Pericardial rupture is a rare diagnosis, usually occurring secondary to high energy blunt force trauma, which can result in subluxation of the heart. Aim: To determine a suitable method for repair of a pericardial defect post traumatic pericardial rupture. Case: We present a 55-year-old male who was found to have a large pericardial defect intra-operatively while undergoing an elective Ross procedure. The defect was repaired with a Gore-Tex membrane. The patient underwent revision sternotomy and repair of the pericardium due to inadequate laxity of the repaired pericardium resulting in hemodynamic instability. Conclusion: Synthetic membranes, such as Gore-Tex membranes, can be used successfully for repair of large pericardial defects but care must be taken to prevent undue tension secondary to taut repair, resulting in hemodynamic compromise.
\end{abstract}

\section{Keywords}

Pericardial Rupture, Pericardium, Trauma, Cardiac Trauma

\section{Introduction}

Pericardial rupture secondary to blunt trauma was first described in the literature in 1864 [1] and is usually associated with high energy incidents, often with a deceleration mechanism and significant mortality [2] [3] [4]. Pericardial rupture post blunt trauma is rare, rendering its diagnosis difficult, with definitive diagnosis often made intra-operatively or on autopsy [3] [4] [5].

We present a patient with a delayed diagnosis of traumatic pericardial rupture resulting in a subluxated heart, found on mediastinal exploration during an elective Ross procedure. This case report demonstrates repair of the rupture using a synthetic membrane and associated complications of cardiac instability if the repair inadvertently restricts cardiac function. 


\section{Case}

A 55-year-old male presents for an elective Ross procedure with moderate to severe aortic regurgitation on a background of congenital bicuspid aortic valve, presenting with symptoms of exertional dyspnea corresponding to NYHA Class III.

Notable in the patient's past medical history were 5 motorbike accidents requiring medical attention spanning from 1996 to 2016. The most significant incident was in 1996 where the patient was ejected over the handlebars of the motorbike at high speeds, sustaining blunt trauma to the chest. His thoracic injuries included right and left sided rib fractures and hemothorax. Due to the age of the incident, medical records could not be sourced, however exploration of the chest was not performed.

Further motorbike accidents in 2002, 2003, 2009 and 2016 resulted in further injuries, requiring interventions including open reduction and internal fixation of the left clavicle. No other intervention was ever done.

Pre-operative workup for the Ross procedure, involving a full heart study (left and right catheterization and coronary angiograms, left ventriculogram, aortogram and oxygen saturations), revealed a dilated root $(43 \mathrm{~mm})$ and ascending aorta $(40 \mathrm{~mm})$ with moderate to severe aortic regurgitation, normal ventricular function, minor coronary artery disease and normal pulmonary artery pressures. Pre-operative chest radiographs from October 2020 showed long standing elevation of the left hemi-diaphragm and bilateral healed rib fractures (Figure 1(A)). This chest radiograph showed minimal change from previous radiographs dating back to January 2011 (Figure 1(B)).

A CT coronary angiogram performed in May 2020 reported an elevated left hemi-diaphragm, causing displacement of the cardiac axis and the heart to lie transversely. The CT showed unremarkable pericardium and pericardial space (Figure 2).

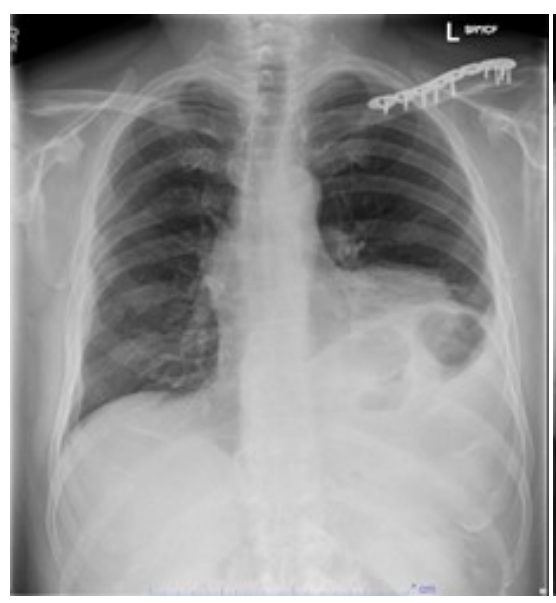

(A)

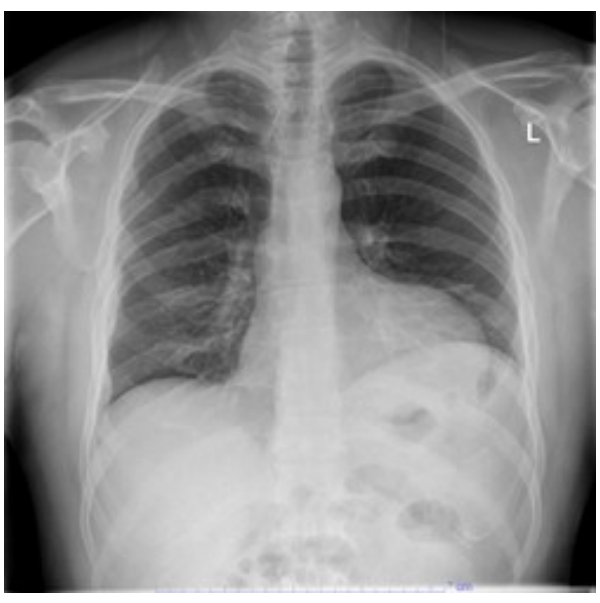

(B)

Figure 1. (A) Chest radiograph from October 2020; (B) Chest radiograph from January 2011. 


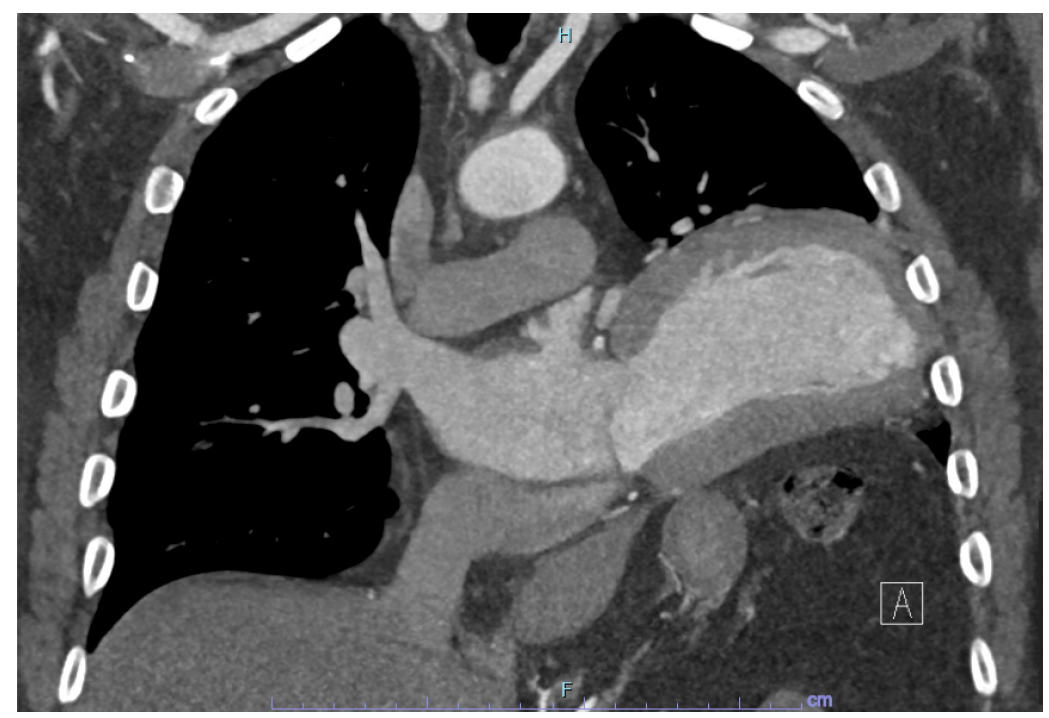

Figure 2. Pre-operative CT chest showing transverse lie of the heart.

Intraoperatively a large left sided defect of the pericardium was found, measuring approximately $15 \mathrm{~cm} \times 10 \mathrm{~cm}$, with complete herniation of the heart into the left thoracic cavity (Figure 3 ); with a raised and fixed hemidiaphragm. The heart was angled transversely and was rotated to the left, with the pulmonary artery laying posterior to the aorta to such a degree that the Ross procedure was aborted due to poor access to the pulmonary valve. The patient instead received a bioprosthetic aortic valve replacement and Dacron grafting of the ascending aorta. The left sided pericardium was shredded and mostly missing, including the portion where the phrenic nerve usually lies. This finding likely explains the raised left hemi-diaphragm. The injury is proposed to have occurred during his previous accidents. No pericardial fluid was found, and the left lung was visible through the vent in the pericardium, and there were no pleural adhesions.

It was difficult to access the aortic valve with the heart in its subluxated position; the heart was thus restored to its normal position. Repair of the pericardium was performed using Gore-Tex membrane sewn in a continuous manner to cover more than $90 \%$ of the defect to oppose the pericardium. Once repaired, the aortic valve became more accessible. Cessation of cardiopulmonary bypass and reperfusion of the heart was uncomplicated, the heart now fixed in its new position. Prior to sternal closure, a new Gore-Tex membrane was used to cover the remaining anterior defect.

Post-operative transesophageal echocardiogram showed a well seated aortic valve replacement with unrestricted motion and no trans-valvular or perivalvular leak.

On the second post-operative day, the patient was noted to have low oxygen saturations ( $88 \%$ - $90 \%$ on 50 liters via nasal prongs), low mixed venous oxygen saturations (38\%) and increased ionotropic supports including $0.25 \mathrm{microg} / \mathrm{kg} / \mathrm{min}$ of milrinone, $150 \mathrm{microg} / \mathrm{min}$ of dobutamine and $200 \mathrm{microg} / \mathrm{min}$ of glyceryl trinitrate. Repeat chest radiographs showed increasing bilateral pleural effusions 


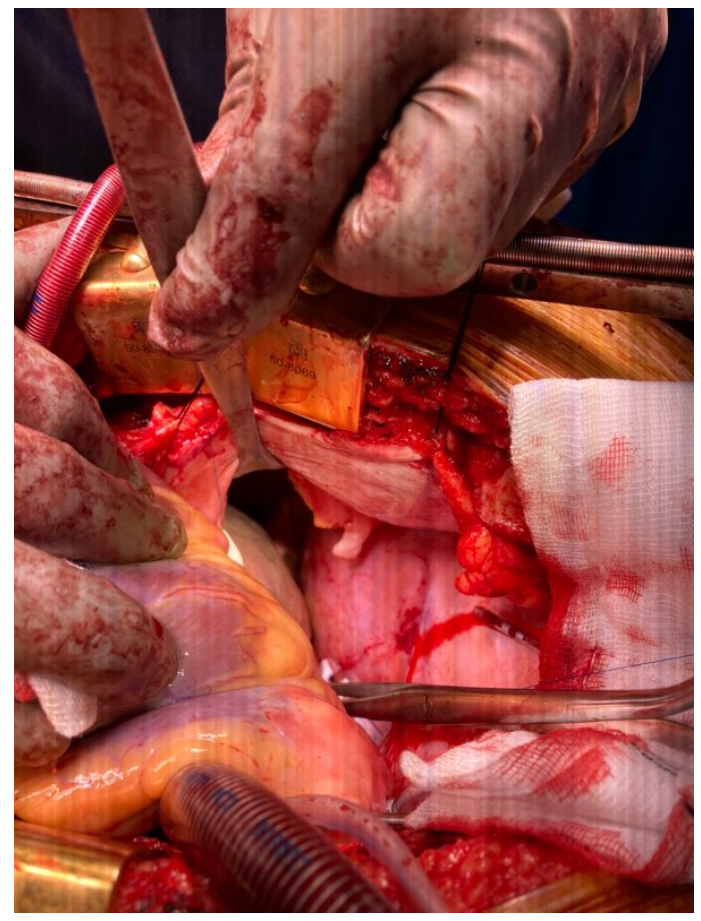

Figure 3. Intraoperative finding of large pericardial defect.

and a left sided consolidation. The decision was made to return the patient to theatre for replacement of the Swan-Ganz catheter and exploration of the pericardial repair, to investigate any surgical repairable cause of his cardiac instability.

On re-sternotomy the right ventricle appeared sluggish to contract and distended. A peri-operative echocardiogram revealed severe right ventricular systolic dysfunction with global hypokinesis, and a pressure loaded right atrium. The previous Gore-Tex membrane was removed with subsequent improvement in right ventricular function, reduction in central venous pressures and reduced ionotropic requirements. A new Gore-Tex membrane was loosely placed anteriorly, without further compromise to cardiac function.

Post-operative chest radiographs revealed a moderate sized left basal pleural effusion and a stable elevation of the left hemidiaphragm, with the heart seated further towards the right side (Figure 4) compared to his pre-operative state (Figure 1(B)). A post-operative cardiac CT with contrast revealed patency of the great vessels, a small pericardial and bilateral pleural effusion with adjacent atelectasis (Figure 5).

Following his second procedure, the patient progressed uneventfully. The patient felt significant improvements in dyspneic symptoms, tolerating greater exertional activity at discharge than pre-operatively. The patient was discharged on day 7 post the re-sternotomy.

Follow up of the patient 10 months post-surgery revealed his symptoms of dyspnea remained modestly improved compared to his pre-operative state. $\mathrm{He}$ had not suffered any further post-operative complications. 


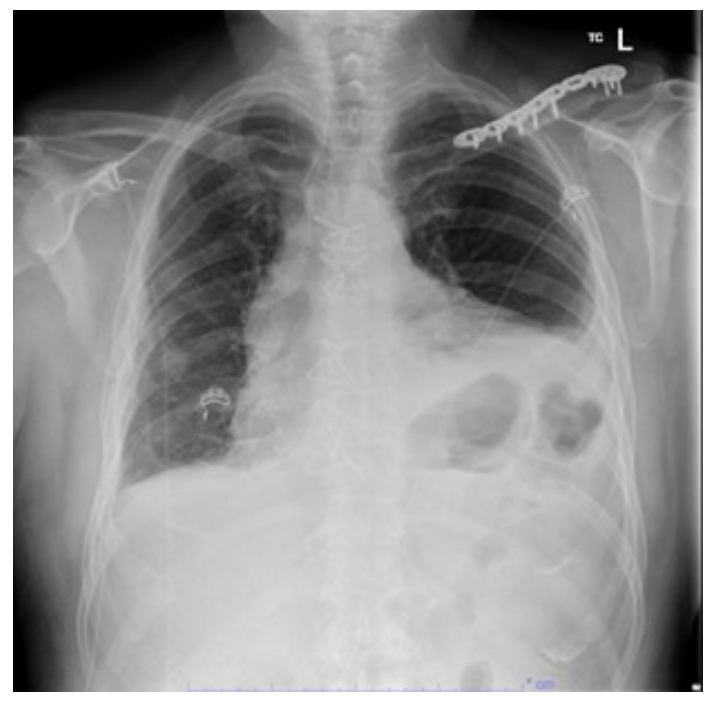

Figure 4. Post-operative chest radiograph taken day 3 post re-sternotomy and Gore-Tex membrane adjustment.

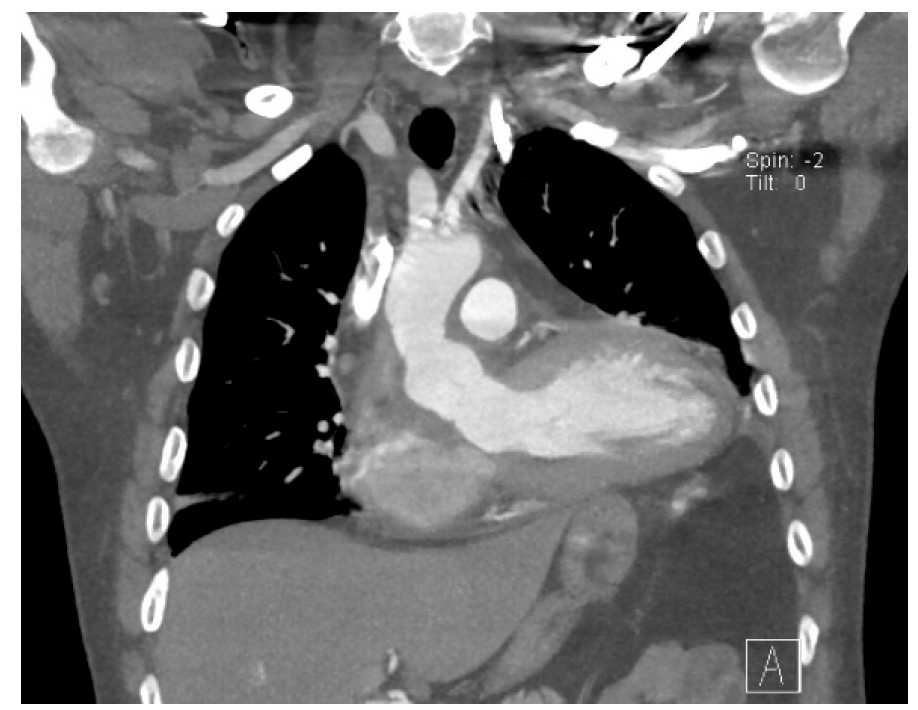

Figure 5. Post-operative CT post re-sternotomy and Gore-Tex membrane adjustment.

\section{Discussion}

Blunt trauma pericardial rupture is commonly left sided [4] and usually presents with symptoms of pericardial tamponade [3] [4]. The exception being a pericardial defect large enough that the fluid is unable to be contained, such as in our patient.

Another concerning feature of pericardial rupture is herniation of the heart through the pericardial defect [3] [4], which has been found to occur with an incidence ranging from $28 \%$ to $64 \%$ of patients with blunt traumatic pericardial rupture [4]. Significant defects can result in complete subluxation of the heart with torsion of the great vessels which may lead to cardiovascular instability [4].

In high energy blunt traumatic thoracic injuries, clinicians should consider pericardial rupture as a possible complication, irrespective of hemodynamic in- 
stability [3] [5], as it can be asymptomatic [5]. Diagnosis may be delayed by a matter of years [4], as demonstrated by our patient.

Recommended investigations include serial chest radiographs as a screening tool to assess cardiac luxation and evolving pathology, electrocardiogram to assess cardiac axis, computed tomography to detect subtle pericardial pathology such as pneumopericardium or pericardial defects [3] [4]. However, as in our case, computed tomography was reported as showing a normal pericardium with no mention of any large defects. Although echocardiography has a low sensitivity for identifying large defects but is very useful in assessing for tamponade and cardiac function in suspected pericardial rupture [4]. Magnetic resonance imaging can also be used to further assess the pericardium if other investigations have been inconclusive [4].

In this case, it is difficult to determine whether the subluxation of the heart was contributing to the patient's symptoms due to the chronicity of the subluxation. Repair of the defect however did facilitate the cardiac surgery.

Repair of the pericardium should be undertaken using sutures for moderate sized defects or synthetic membranes for larger defects [3] [4], such as Gore-Tex membrane as in our case. The repaired membrane should prevent cardiac luxation and further hemodynamic compromise due to torsion of the great vessels [3]. Small defects may be left alone if cardiac herniation is deemed unlikely [4]. Too tight a repair is to be avoided in chronic cases with cardiac subluxation as the heart would have adjusted to its new position, as in our case. There should remain adequate space in the post-repair position to avoid post-operative hemodynamic instability.

\section{Conclusion}

Pericardial rupture is a rare diagnosis, most commonly secondary to blunt high force chest trauma. Defects in the pericardium may result in subluxation of the heart. Repair of larger pericardial defects may be undertaken using synthetic membranes such as the Gore-Tex membrane to restore anatomy, but care must be taken to prevent undue tension secondary to taut repair resulting in hemodynamic compromise.

\section{Ethics Approval and Informed Consent}

This article does not contain any studies involving human participants or animals performed by the author. Informed consent was obtained from the patient in the study.

\section{Conflicts of Interest}

The authors declare that they have no competing interest.

\section{References}

[1] Morel-Lavallee, V.A. (1864) Rupture du pericarde; bruit de roue hydraulique; bruit 
de moulin. Gaz Med Paris, 19, 695.

[2] Fulda, G., Rodriguez, A., Turney, S.Z. and Cowley, R.A. (1990) Blunt Traumatic Pericardial Rupture. A Ten-Year Experience 1979 to 1989. The Journal of Cardiovascular Surgery, 31, 525-530.

[3] Motto, D.A., Kurapati, S., Atencio, D.C., Miller, M.A. and Stahlfeld, K.R. (2018) Pericardial Rupture with Intermittent Cardiac Luxation. Texas Heart Institute Journal, 45, 115-116. https://doi.org/10.14503/THIJ-16-5803

[4] Sherren, P.B., Galloway, R. and Healy, M. (2009) Blunt Traumatic Pericardial Rupture and Cardiac Herniation with a Penetrating Twist: Two Case Reports. Scandinavian Journal of Trauma, Resuscitation and Emergency Medicine, 17, Article No. 64. https://doi.org/10.1186/1757-7241-17-64

[5] Khalpey, Z., Rajab, T.K., Schmitto, J.D. and Camp, P.C. (2011) Traumatic Pericardial Rupture with Skeletonized Phrenic Nerve. Journal of Cardiothoracic Surgery, 6, Article No. 6. https://doi.org/10.1186/1749-8090-6-6 